\title{
New Dimensions of Pharmaceutical Analysis
}

\section{Victoria F Samanidou*}

\section{Department of Analytical Chemistry, Aristotle University of Thessaloniki, Greece}

Over the past several decades, the analytical techniques pertaining to drug detection and characterization have evolved a great deal resulting in development, optimization and validation of vast array of methodologies and techniques. Continued efforts are being made for impurity profiling while bringing in better accuracy and precision for separation and quantification of the analytes. Pharmaceutica Analytica Acta reports these advances periodically. The current issue of the journal focuses on distribution of nasal spray droplet distribution, in silico studies on Spebrutinib and pharmaceutical analysis based on flow analysis techniques.

When compared to other topical application of therapeutic agents, nasal spray is regarded as effective for rapid drug delivery and efficacy due to rich nasal vascularization and close proximity of olfactory region to the central nervous system. However, the droplet size distribution plays an important role in the safety of administration and its prevention from entering the tracheo-bronchi system. Rao et al. [1] have conducted analysis of nasal spray droplet size distribution and determined the effect of different actuation angle on the observations. The study revealed that velocity does not determine the droplet size though change in actuation angle did. The study emphasized that the method was precise, robust and has capacity to distinguish between different products. The developed method has immense relevance for evaluation of commercial nasal spray quality.

In silico studies are conducted in order to evaluate the efficacy of different drug analogues and their docking properties. Spebrutinib is a potential anti-cancerous agent as it effectively inhibits tyrosine kinase, an enzyme important for cancer cell survival. Al-Obaidi et al. [2] have conducted in silico studies using different analogues of this anti-cancerous agent. The design and docking studies were also useful for synthesis and chemical characterization of the analogues.
The study also emphasized on conducting biological activity against cancerous cell and toxicity assessment in normal cells.

The techniques of separation and quantification of the molecular entities play major role in pharmaceutical analyses. After deliberating about the technological advancements, advantages and the limitation of hyphenated approaches, Economou [3] has emphasized that liquid separation based hyphenation of flow analysis serves as complementary to customary liquid chromatography and has immense scope in pharmaceutical analysis with capacity to determine large number of pharmaceuticals including application for low to medium pressure separations and development of gradient elution protocol as well as online sample pretreatment.

The articles in the current issue are of immense significance in developing, modifying, optimizing and validation of better analytical strategies and approaches having relevance to nasal spray based therapy; designing and developing precise drug analogues for cancer treatment as well as separation of new pharmaceutical analytes which pose substantial analytical challenges.

\section{REFERENCES}

1. Ravi Prasada RM, Mogadati P, Arutla S, Senthi M. Development and Validation of Robust Analytical Method to Determine Droplets Size Distribution of Nasal Spray Using Laser Diffraction Technique. Pharm Anal Acta. 2019; 10:611.

2. Al-Obaidi ZMJ, Rasheed OFA, Mahdi MF, Raauf AMR. In Silico Design, Synthesis and Characterization of New Spebrutinib Analogues. Pharm Anal Acta. 2019;10:612.

3. Economou A. Advances in the Hyphenation of Flow Analysis Techniques with Liquid Separations for Pharmaceutical Analysis. Pharm Anal Acta. 2019;10:613.

"Correspondence to: Victoria F. Samanidou, Department of Analytical Chemistry, Aristotle University of Thessaloniki, Greece, Tel: +302310997698; Fax: +302310997719; E-mail: samanidu@chem.auth.gr

Received: September 19, 2019; Accepted: September 23, 2019; Published: September 30, 2019

Citation: Samanidou VF (2019) New Dimensions of Pharmaceutical Analysis. Pharm Anal Acta 10:614. doi: 10.35248/2153-2435.19.10.614

Copyright: (C) 2019 Samanidou VF. This is an open-access article distributed under the terms of the Creative Commons Attribution License, which permits unrestricted use, distribution, and reproduction in any medium, provided the original author and source are credited. 